\title{
Laryngeal Crohn's Disease: Case Report and Review of Literature
}

\author{
Samia A Fawaz ${ }^{1 *}$, Ahmed El-Demerdash ${ }^{2}$ and Manal I Salman ${ }^{3}$
}

${ }^{1}$ Otorhinolaryngology, Ain Shams University, Egypt

${ }^{2}$ Phoniatric Unit, Military Academy, Egypt

${ }^{3}$ Pathology department, Ain Shams University, Egypt

\begin{abstract}
Introduction: Crohn's disease (CD) is classically described as a chronic granulomatous inflammatory bowel disease, laryngeal and other otolaryngologic manifestations of CD are uncommon and may be subtle, as laryngeal involvement by CD has been reported in only six cases in the medical literature.

Objective: we describe a case of an Egyptian man with extra intestinal CD that involves the supraglottic and subglottic regions, but with skipping of the glottis.

Conclusion: This case demonstrates the need to be oriented with extra intestinal CD especially if the lesion was mainly laryngeal, and therefore, a high degree of suspicion is necessary to diagnose the disease, as early recognition is imperative, and we recommend a detailed study on a large number of patients with inflammatory bowel disease (IBD) and CD on the long term effect of colectomy on development of extra intestinal manifestations, as we suspect that colectomy might have triggered the onset of airway involvement many years later.
\end{abstract}

Keywords: Crohn's disease; Inflammatory bowel disease; Laryngeal manifestations; Steroid resistant Crohn's disease

\section{Introduction}

Crohn's disease (CD) is classically described as a chronic granulomatous inflammatory bowel disease. Its pathologic findings include noncontiguous chronic inflammation and non caseating granulomas. Any segment of the gastrointestinal tract (GIT) can be involved in the disease process, but it is unusual for the disease to extend into the upper aerodigestive system [1].

It is associated with a unique set of potential intra luminal and extra luminal complications that can have markedly different courses in individual patients. The intraluminal manifestations are well-known by clinicians and will not be the focus of this review [2].

The most common extra intestinal manifestations are central and peripheral arthritis, hepatobiliary disease, cutaneous and ocular manifestations [3]. However, laryngeal and other otolaryngologic manifestations of CD are uncommon and may be subtle [4].

Here, we describe a case of an Egyptian man with extra intestinal CD that was marked by quiescent involvement of the lower gastrointestinal tract and florid involvement of the supraglottic and subglottic regions, but with skipping of the glottis.

\section{Case Report}

A 48-year-old man presented to Otorhinolaryngology department, Ain Shams University hospital in November 2011, complaining of failure of decanulation from tracheostomy, which was performed 2 years ago, his condition started since adulthood, as he has had longlasting GIT symptoms since 1984, he has had bouts of urgency with incontinence as well as intermittent abdominal pain, at that time he was diagnosed with a possible ulcer versus appendicitis due to severe abdominal pain, then laparotomy was performed, at which point multiple small bowel lesions were found with pathology consistent with CD. Then resection of the diseased small bowel was performed, after that, he was on postoperative medication and ended up having significant diarrhea up to 25 times per day, he suffered from malabsorption and weight loss for many years afterward.
In 1985, a recurrence of the CD at the surgical anastmosis occurred, a surgeon started him on Questran ${ }^{\circledR}$ for few years, hence the patient improved, but in 1995, his symptoms had returned with diarrhea and mild pain, with no history of specific medications at that point.

In the years 2005 through 2006, he received medication in the form of Pentasa ${ }^{\circledR}$, Imuran ${ }^{\circledR}$ for varying amounts of time to control his GIT symptoms

In 2009, he started to complain of symptoms sounds Obstructive Sleep Apnea, Uvulopalatopharyngoplasty (UPPP) was performed, but he had ongoing symptoms in spite of this; in addition he had some symptoms of dysphagia on occasion for both solid and liquids. On examination, there was mild stridor at rest, his voice was slightly muffled as well, flexible nasopharyngoscopy with stroboscopy was performed, showed extensive supraglottic edema involving the aryepiglottic folds, mucosa over the cricoarytenoid joints and the epiglottis

At that time, blood work for sarcoidosis work up was done, which was negative, and the patient was placed on pulse dose of Prednisone for two weeks only, hence the patient noticed mild improvement of his symptoms during day, but he still complained at night, then flexible endoscopy was repeated; revealed mildly decreased edema particularly on the right aryepiglottic fold, an awake tracheostomy was performed, and a microlaryngoscopy with $\mathrm{CO}_{2}$ Laser vaporization of left aryepiglottic fold with biopsies from left aryepiglottic fold and redundant mucosa over arytenoids; which showed numerous non necrotizing granulomatous inflammation with fibrosis, edema and lymphangiectasia, many of the granulomas centered on or within

*Corresponding author: Samia A Fawaz, Otorhinolaryngology, Ain Shams University, Egypt, E-mail: samyafawaz@gmail.com

Received December 14, 2011; Accepted December 23, 2011; Published December 28, 2011

Citation: Fawaz SA, El-Demerdash A, Salman MI (2012) Laryngeal Crohn's Disease: Case Report and Review of Literature. J Clinic Case Reports 2:e107. doi:10.4172/2165-7920.1000e107

Copyright: ( 2012 Fawaz SA, et al. This is an open-access article distributed under the terms of the Creative Commons Attribution License, which permits unrestricted use, distribution, and reproduction in any medium, provided the original author and source are credited. 
lymphatics (favors laryngeal CD) (Figures 1 and 2), in addition his septic and lymphoma work up were negative.

Laboratory work showed $\mathrm{Na}=138, \mathrm{Cl}=102$, urea $=1.1$, normal creatinine, normal $\mathrm{Ca}$, Phosphrus $=1.2, \mathrm{Mg}=0.68$, total bilirubin $=$ 11, alk Phosphatase $=83, \mathrm{ALT}=19, \mathrm{AST}=17, \mathrm{LD}=116, \mathrm{CBC}$ showed; $\mathrm{WBCs}=4.6, \mathrm{Hb}=12.3, \mathrm{MCV}=84.3$, Platelets $=274, \mathrm{ESR}=16, \mathrm{CRP}=5.2$.

Six weeks later another session of Laser vaporization of the redundant edematous left aryepiglottic fold was done, and the pathological assessment was repeated and revealed the same previous findings, special stains for fungi and Acid fast bacilli were performed and no organisms were identified, in addition to negative tuberculin test or PPD test., with negative serology for Q fever and brucellosis.

After that flexible nasopharyngoscopy was performed and it showed much improvement of supraglottic larynx, particularly on left side, but he can't cork the tracheostomy at night, so in 2010, a $3^{\text {rd }}$ procedure was done ( $\mathrm{CO}_{2}$ Laser partial supraglottic laryngectomy) mainly of right side, followed by another colonoscopy that revealed clearly inflamed mucosa with several discrete ulcers at area of previous anastmosis, with random biopsies taken to be examined pathologically, which revealed focal congestion and hemorrhage with increase in lymphocytic infiltrate in the lamina propria, without evidence of active colitis. Upper GIT endoscopy showed only mild duodenitis.

Two months later, nasopharyngoscopy was done, revealed redundant mucosa over the right arytenoids and post cricoids region (which was not present previously) that herniating into the posterior larynx, so a $4^{\text {th }}$ session of Laser was done to excise the redundant tissue, and four weeks later a $5^{\text {th }}$ session was done for excision of recurrent redundant postcricoid mucosa, which was indrawing over the posterior larynx on inspiration. Then the patient was placed on a regimen consisted of imuran 150mg PO daily, Calcium, Vitamin D, and Rimacade ${ }^{\circledR}$.

In 2011, flexible nasopharyngoscopy performed at Kobri El Koba Military Hospital showed cobble stone appearance of the nasopharyngeal side of the soft palate, in addition to the redundant tissue over the right aryepiglottic fold and over right arytenoids, but with much improvement of left aryepiglottic fold (Figure 3).Overall ,

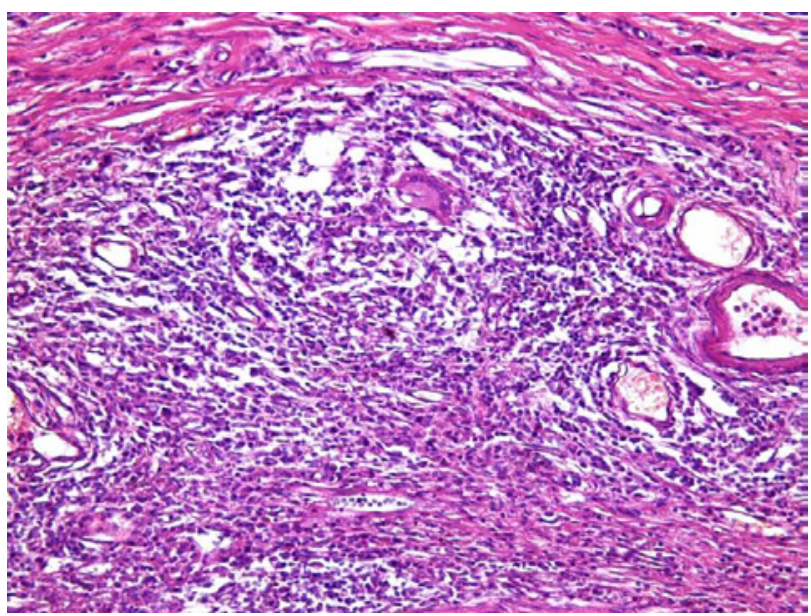

Figure 1: Biopsy examined from the supraglottic swelling showing; numerous non necrotizing granulomas with fibrosis and lymphangiectasia (X 200).

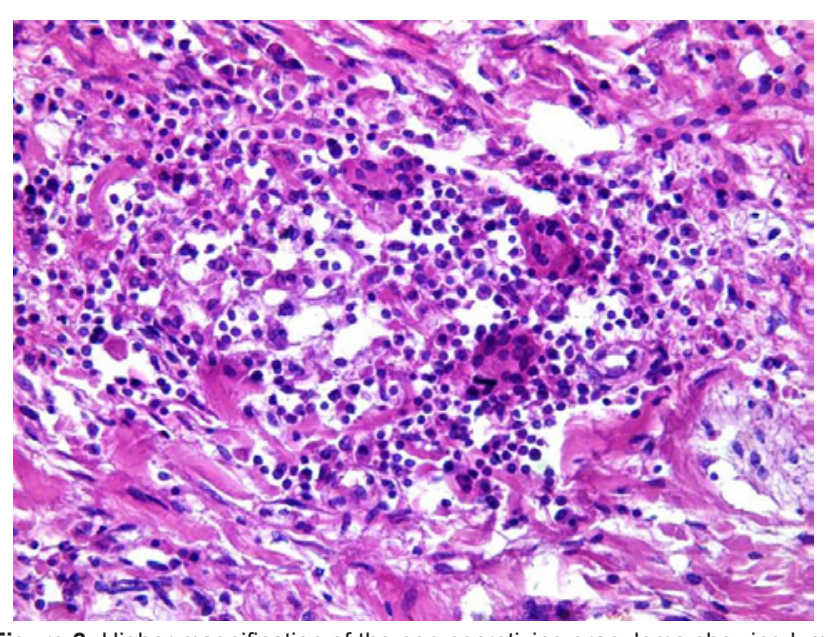

Figure 2: Higher magnification of the non necrotizing granuloma showing lymphocytes, plasma cells and multinucleated giant cells. (X400).

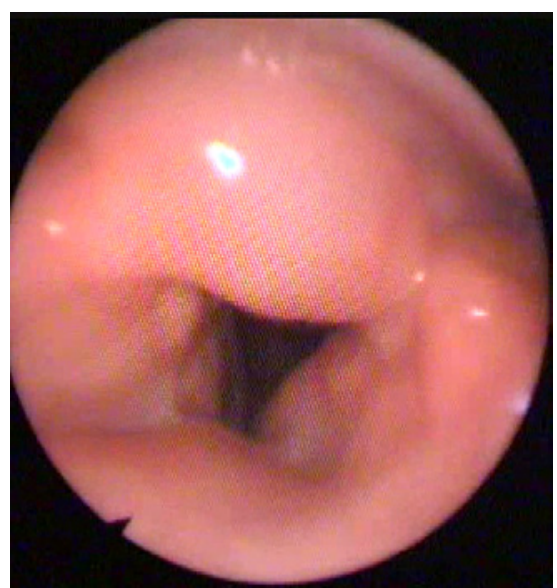

Figure 3: Flexible nasopharyngoscopy showed redundant tissue over the right aryepiglottic fold and over right arytenoids, but with a much improvement of left aryepiglottic fold.

however, he certainly has had an adequate supraglottic airway at rest and when awake, but by advancing the fibroptic laryngoscopy down to the level of tracheostomy, it revealed subglottic circumferential narrowing by granulomatous swelling (Figure 4), and this was the cause hindering the decanulation which was further needed after controlling of the activity of the systemic disease, but with fear from its recurrence or the development of sever stenosis if Laser excision was done, then the patient was placed on a regimen consisted of local intralesional injection of steroid (Kenacort A 40mg) twice with two weeks interval, and Methotrexate (25mg IM every 2 weeks), this regimen revealed moderate improvement so the patient continued on this regimen .

\section{Discussion}

Crohn's disease is a well-known inflammatory bowel disease (IBD) of unknown etiology marked by relapsing and remitting granulomatous inflammation of the alimentary tract [4].

Extra intestinal manifestations of Crohn's disease (CD) may appear anytime during the course of the disease process and may be the initial presentation. Findings are nonspecific, primarily edema and 


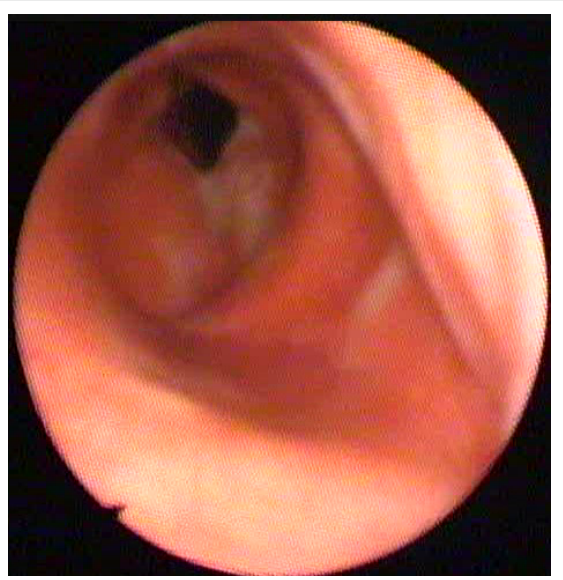

Figure 4: Flexible nasopharyngoscopy showed subglottic circumferential narrowing by granulomatous swelling .

ulcerations, and may be confused with a multitude of other disease processes. Awareness of these manifestations in the head and neck will prevent misdiagnosis or a delay in diagnosis [4].

The extra intestinal involvement of $\mathrm{CD}$ is becoming more readily identified [5], it has been reported in $36 \%$ of patients with CD [1]. And more than $40 \%$ of them experience cutaneous manifestations. However, involvement of the aerodigestive tract has been documented in the literature, and oral manifestations of $\mathrm{CD}$ have been noted to precede its gastrointestinal appearance in 26 to $60 \%$ of cases [1]. Recurrent aphthous ulcer is the most common oral manifestation of inflammatory bowel disease (IBD) and is a frequent finding in CD. The other disease-specific oral manifestations include mucosal tags, lip swelling, fissures, buccal mucosal swelling or cobble stoning, deep linear ulcerations and localized mucogingivitis [6] but, involvement of the esophagus has been reported to occur in fewer than $2 \%$ of cases [1].

Although historically considered a relatively rare phenomenon, there is a wide variety of pulmonary symptoms that occur with IBD and $\mathrm{CD}$ [3], the varied symptomatology is related to the location of pulmonary involvement, as it may affect the upper airways, the lung parenchyma, and pulmonary vasculature. Pulmonary involvement is even more prevalent when considering persons with subclinical disease. While overt pulmonary symptoms may be uncommon, abnormalities on pulmonary function testing have been noted in $>60 \%$ of those with CD [7]. IBD can affect virtually every part of the airway, patients typically present with hoarseness and cough early in the course of their bowel disease; however, there is often a significant delay in diagnosis. There is usually a good response to inhaled corticosteroids, although laser ablation has been used in refractory cases [8].

However, laryngeal involvement by $\mathrm{CD}$ has been reported in only six cases in the medical literature [5]. Here we describe the $7^{\text {th }}$ case of laryngeal Crohn's disease, as our case revealed the presence of supraglottic edematous tissue that involved the epiglottis and aryepiglottic folds, the mucosa over arytenoids, and lastly the post cricoid and subglottic regions. Repeated biopsies have shown a granulomatous process suggestive of Crohn's (considering his past history and excluding other suspected diseases as sarcoidosis, lymphoma, tuberculosis, fungal granulomas, $\mathrm{Q}$ fever and brucellosis)

Corticosteroid drugs are the mainstay treatment of $\mathrm{CD}$ related airway involvement, the route, dosage, titration and duration of treatment vary in different patients and are largely empirical. Oral steroids are more readily efficacious, enabling quicker control of symptoms and are indicated in patients with moderate or severe airway involvement [9] but it may have transient or imperceptible effects, and in some patients the illness is refractory to any form of therapy [10].

The other medical options of CD consist of 5-aminosalicylic acid (5ASA) with or without steroids depending on the activity of the disease. Immunosuppressive drugs like azathioprine, 6 mercaptopurine, cyclosporin A and methotrexate are used when there is failure of response to corticosteroids [6]. In 2003, Ottaviani reported a rare case of extra-intestinal CD resistant to steroid therapy, which was successfully treated with infliximab, a chimeric antibody directed against TNF-alpha that is the only registered agent for the treatment of CD [11].

On the contrary to the previous studies and to Croft and Wilkinson [12], who reported a case of laryngeal involvement of CD that resolved with the use of oral steroids and also Kelly et al. [15] who reported two cases of Crohn's involvement of the larynx, both of which improved with oral steroids, as well as Ottaviani [13] who reported that Oral and laryngeal involvement were treated successfully by steroids [11]. Our case showed only very mild improvement of the laryngeal edema on the standard medical management with systemic steroid therapy (short course therapy) and Imuran, which may be attributed to the extensive involvement of the larynx, therefore, he has had multiple interventional procedures for improving the airway, as much improvement occurred by Laser surgical excision (this was the typical way the patient responded as the area that operated upon typically was better), but with relapse at other sites, with the most favorable results attributed to intralesional steroid injection and Methotrexate .

But in accordance to our results of failed standard medical management, Ulnick and Perkins found that the aggressive laryngeal form of $\mathrm{CD}$ has failed to respond to standard medical management with systemic and topical steroids and 6-mercaptopurine. Even with surgical intervention, they have only been able to slow the process. Since performing surgery and increasing the 6-mercaptopurine dosage, they have been able to lengthen the interval between the patient's laser treatments to every 9 to 12 months. Even so, they were significantly concerned about the progressive laryngeal involvement, which remained a difficult management problem [1]. This very aggressive manifestation of $\mathrm{CD}$ can be classified technically as metastatic CD because of its intestinal and extra intestinal involvement [14].

As our patient developed air way symptoms 25 years after colectomy, which is in accordance to Kelly et al. [15] who found that in approximately $7 \%$ of IBD patients who developed airway involvement, the onset of respiratory symptoms was weeks to years after the development of clinically confirmed IBD, and added that post colectomy patients are not immune to the development of airway involvement (which may be severe) and colectomy may be a risk factor for onset and progression of severe airway involvement

\section{Conclusion}

This case demonstrates the need to be oriented with extra intestinal Crohn's disease especially if the lesion was mainly laryngeal, and therefore, a high degree of suspicion is necessary to diagnose the disease, as early recognition is imperative, to be proactive in the 
Citation: Fawaz SA, El-Demerdash A, Salman MI (2012) Laryngeal Crohn's Disease: Case Report and Review of Literature. J Clinic Case Reports 2:e107. doi:10.4172/2165-7920.1000e107

management of these patients. , and we recommend a detailed study on large number of patients with IBD and CD on the long term effect of colectomy on development of extra intestinal manifestation, as we suspect that colectomy may trigger the onset of airway involvement many years later.

\section{References}

1. Ulnick KM, Perkins J (2001) Extra intestinal Crohn's disease: Case report and review of the literature. Ear Nose Throat J 80: 97-100.

2. Bodger K (2005) Economic implications of biological therapies for Crohn's disease: review of infliximab. Pharmacoeconomics 23: 875-888.

3. Brian R Zeno (2007) Pulmonary Manifestations of Inflammatory Bowel Disease.

4. Yang J, Maronian N, Reyes V, Waugh P, Brentnall T, et al. (2002) Laryngeal and Other Otolaryngologic Manifestations of Crohn's Disease. J Voice 16: 278282.

5. Gianoli GJ, Miller RH (1994) Crohn's disease of the larynx. J Laryngol Otol 108: 596-598.

6. Ganesh R, Suresh N, Ezhilarasi S, Rajajee S, Sathiyasekaran M (2006) Crohn's Disease Presenting as Palatal Ulcer. Indian J Pediatr 73: 229-231.

7. Songur N, Songur Y, Tuzun M, Dogan I, Tuzun D, et al. (2003) Pulmonary function tests and high-resolution $\mathrm{CT}$ in the detection of pulmonary involvement in inflammatory bowel disease. J Clin Gastroenterol 37:292-298.

8. Janssen WJ, Bierig LN, Beuther DA, Miller YE (2006) Stridor in a 47-year-old man with inflammatory bowel disease. Chest 129: 1100-1106.

9. SpiraA, GrossmanR, BalterM (1998) Large airway disease associated with inflammatory bowel disease. Chest 113: 1723-1726.

10. Camus Ph, Colby TV (2011) Bronchiectasis associated with inflammatory bowel disease. Bronchiectasis by Floto RA and Haworth CS (editors), Eur Resp Soc Monograph 52: 163-177.

11. Croft CB, Wilkinson AR (1972) Ulceration of the mouth, pharynx, and larynx in Crohn's disease of the intestine. Br J Surg 59: 249-252.

12. Kelly JH, Montgomery WW, Goodman ML, Mulvaney TJ (1979) Upper airway obstruction associated with regional enteritis. Ann Otol Rhinol Laryngol 88: 9599.

13. Ottaviani F, Schindler A, Capaccio P, Petrone M, Bianchi Porro G (2003) New therapy for orolaryngeal manifestations of Crohn's disease. Ann Otol Rhino Laryngol 112: 37-39.

14. Chen W, Blume-Peytavi U, Goerdt S, Orfanos CE (1996) Metastatic Crohn's disease of the face. J Am Acad Dermatol 35: 986-988.

15. Kelly MG, Frizelle FA, Thorndey PT, Beckert L, Epton M, et al. (2006) Inflammatory bowel disease and the lung. Is there a link between surgery and bronchiectasis? Int J Colorectal Dis 21: 754-757. 